\title{
4DCT Scanning Technique for Primary Hyperparathyroidism: A Scoping Review
}

\author{
Steven Raeymaeckers $\mathbb{D D}^{1},{ }^{1}$ Maurizio Tosi ${ }^{1},{ }^{1}$ and Johan De Mey $\mathbb{D i D}^{1,2}$ \\ ${ }^{1}$ Universitair Ziekenhuis Brussel, Laarbeeklaan 101, Jette 1090, Belgium \\ ${ }^{2}$ Radiology, Vrije Universiteit Brussel, Laarbeeklaan 103, Jette 1090, Belgium \\ Correspondence should be addressed to Steven Raeymaeckers; steven.raeymaeckers@uzbrussel.be
}

Received 2 January 2021; Revised 11 April 2021; Accepted 13 May 2021; Published 21 May 2021

Academic Editor: Lorenzo Faggioni

Copyright (c) 2021 Steven Raeymaeckers et al. This is an open access article distributed under the Creative Commons Attribution License, which permits unrestricted use, distribution, and reproduction in any medium, provided the original work is properly cited.

\begin{abstract}
Objective. 4DCT for the detection of (an) enlarged parathyroid(s) is a commonly performed examination in the management of primary hyperparathyroidism. Protocols are often institution-specific; this review aims to summarize the different protocols and explore the reported sensitivity and specificity of different 4DCT protocols as well as the associated dose. Materials and Methods. A literature study was independently conducted by two radiologists from April 2020 until May 2020 using the Medical Literature Analysis and Retrieval System Online (MEDLINE) database. Articles were screened and assessed for eligibility. From eligible studies, data were extracted to summarize different parameters of the scanning protocol and observed diagnostic attributes. Results. A total of 51 articles were included and 56 scanning protocols were identified. Most protocols use three $(n=25)$ or four different phases $(n=23)$. Almost all authors include noncontrast enhanced imaging and an arterial phase. Arterial images are usually obtained 25-30 s after administration of contrast, and less agreement exists concerning the timing of the venous phase(s). A mean contrast bolus of $100 \mathrm{~mL}$ is administered at 3-4 mL/s. Bolus tracking is not often used $(n=3)$. A wide range of effective doses are reported, up to $28 \mathrm{mSv}$. A mean sensitivity of $81.5 \%$ and a mean specificity of $86 \%$ are reported. Conclusion. Many different 4DCT scanning protocols for the detection of parathyroid adenomas exist in the literature. The number of phases does not appear to affect sensitivity or specificity. A triphasic approach, however, seems preferable, as three patterns of enhancement of parathyroid adenomas are described. Bolus tracking could help to reduce the variability of enhancement. Sensitivity and specificity also do not appear to be affected by other scan parameters like tube voltage or tube current. To keep the effective dose within limits, scanning at a lower fixed tube current seems preferable. Lowering tube voltage from $120 \mathrm{kV}$ to $100 \mathrm{kV}$ may yield similar image contrast but would also help lower the dose.
\end{abstract}

\section{Introduction}

Primary hyperparathyroidism is a common endocrine disease. In the case of an asymptomatic patient over the age of 50 without end-organ complications, conservative treatment can be assumed $[1,2]$. The only cure for the disease is surgery, with resection of the affected gland(s). Bilateral exploration of the neck is the historical standard for treatment. In the last decades, however, a minimally invasive surgical approach has been made possible by more effective means of preoperative imaging combined with the development of rapid parathyroid hormone determination techniques allowing for intraoperative PTH monitoring [3].
The most accessible diagnostic technique is ultrasound because it is widely available at low cost, and it presents no adverse effects [4]. As a bonus, ultrasound is the preferred method of examination for the thyroid gland. This way parathyroid lesions can be differentiated from thyroid nodules and other thyroid pathologies. The sensitivity is very operator-dependent but can be as high as $84 \%$ in hands of an experienced ultrasonographer [5]. Color Doppler can be used to differentiate parathyroid lesions from other cervical masses, such as lymph nodes and thyroid nodules [6]. Small lesions $(<5 \mathrm{~mm})$ can be difficult to detect. False-negative results can occur, especially in the case of ectopic glands or in the presence of a large thyroid goiter [7]. 
Scintigraphy has the highest sensitivity compared to the other techniques: $88-90 \%$ and even higher sensitivity when combined with SPECT/SPECT-CT, the latter providing useful anatomical detail [8]. This technique is the method of choice when an ectopic localization is suspected or in patients having undergone prior neck surgery. The procedure relies on the uptake of $99 \mathrm{mTechnetium}(99 \mathrm{mTc})$-sestamibi in the overactivated mitochondria-rich oxyphil cells of the parathyroid gland and the difference in the rate of washout of this tracer with the thyroid. To overcome false positive results deriving from the uptake of sestamibi in solid thyroid nodules, another tracer can be administered that is exclusively taken up by the thyroid tissue, thereby permitting a subtraction of the thyroid. This method is known as the double-tracer technique and can achieve a specificity of over $90 \%$ [9].

MRI also allows for the evaluation of parathyroid disease. This technique, like ultrasound, is free of ionizing radiation but it is far a less available modality (a problem also found with scintigraphy). Due to the lack of ionizing radiation, MRI can be used without hesitation to detect ectopic glands. On a 1.5 Tesla system, the reported sensitivity of this technique is $80 \%$; assumedly a better visualization on 3 Tesla systems can be obtained [10-12].

Computed tomography (CT) of the neck without contrast is of no value since it is not possible to discern between parathyroid tissue, ectopic thyroid tissue, and lymph nodes. Four-dimensional CT (4DCT) combines three-dimensional imaging with the inclusion of time as the fourth dimension: this allows for an evaluation of the pattern of enhancement of lesions over time. By means of the evaluation of enhancement, abnormal parathyroid glands can be detected with a sensitivity of $85.7 \%$ : typical parathyroid adenomas are hypoattenuating to thyroid tissue on noncontrast enhanced imaging (NECT) and demonstrate avid arterial hyperenhancement during the arterial phase as well as rapid washout on the venous phase $[13,14]$. 4 DCT is also considered to be a useful technique in the case of ectopic glands or in the case of persistence/recurrence after the initial surgery.

Many different study protocols have been suggested by different authors over the past 15 years; protocols are often very institution-specific. We set out to examine the literature to review the different protocols and their reported sensitivity/specificity. The number of different phases used in a protocol as well as their timing could be a defining factor in sensitivity: as more phases are available, it could be argued that this would increase the sensitivity of the examination. Factors like tube current, tube voltage, and administration of contrast medium affect image noise and contrast and could therefore also have implications toward sensitivity. These factors also influence the effective dose, a key part to consider in multiphasic studies using ionizing radiation.

\section{Materials and Methods}

2.1. Research Question. This scoping review seeks to answer a multivalent research question. First, we want to establish the number of phases and their timing as used by different authors in the literature. Secondly, we wish to assess whether different protocols are associated with different sensitivity or specificity. We also look at other scanning protocol factors like tube current and tube voltage, contrast bolus volume, and timing: factors that not only can affect the sensitivity of the exam but also influence the effective dose, a key part to consider in multiphasic studies using ionizing radiation.

2.2. Search Strategy. This scoping review is reported according to the PRISMA Extension for Scoping Reviews (PRISMA-ScR) Checklist. A literature search was independently conducted by two radiologists using the MEDLINE database from April 2020 until May 2020 using the terms "4DCT" and "parathyroid."

2.3. Inclusion and Exclusion Criteria. We included all peerreviewed literature written in English or French. There was no limitation on the publication date. Exclusion criteria were articles written in a language other than English or French.

2.4. Data Selection and Charting Process. The two investigators independently screened the MEDLINE database for relevant articles. A first selection was made based on the title and abstract. Next, each investigator screened the obtained articles for relevancy, and a second selection was made. Finally, the remaining articles underwent a final screening: only studies citing information about the number of phases used in the scanning protocol were included in the scoping review. Forty articles were not relevant. Information regarding publication details (e.g., author, publication date) and study details (e.g., study design, scanning protocol) was obtained. Data was managed in Excel (Microsoft, Redmond, USA). Several scanning protocol factors were included in this review. We checked for the presence of a NECT in the study protocol. We evaluated the number of obtained phases and their timing: we defined a subdivision in an arterial phase (earlier than 40 seconds after contrast administration), a venous phase (40 seconds-70 seconds), a delayed venous phase (70 seconds-100 seconds), and a very delayed phase (later than 100 seconds). We evaluated whether this timing was expressed in absolute or relative relation to the onset of contrast administration; we expressed (where possible) all times in absolute relation to the administration of contrast. We also checked for the use of bolus tracking, which is defined as the use of a continuous density measurement in an arterial structure (usually at the level of the aortic arch) prior to obtaining the first arterial phase.

We also included other parameters of the scanning protocol: tube current is determined by the rate at which $\mathrm{X}$-rays are produced in the X-ray tube (i.e., photons per second). It is expressed in milliampere ( $\mathrm{mA})$. Tube voltage is a parameter in direct relation to the number of $\mathrm{X}$-rays produced. It is expressed in kilovolt $(\mathrm{kV})$. The volume of the contrast bolus was included, as well as the injection speed expressed in $\mathrm{mL} / \mathrm{s}$. We included the effective dose, expressed in millisievert $(\mathrm{mSv})$. Lastly, we included the reported sensitivity, PPV, specificity, and other related data provided. 


\section{Results}

3.1. Identification of Potential Studies. The searches from the MEDLINE database hit a total of 91 records that were screened after the removal of duplicates. All articles were available to us in full-text format. The full-text screening stage led to 91 potential articles relevant to our scoping review. Additional articles were excluded after full-text assessment for the reasons mentioned in "Materials and Methods". A flow chart is provided in Figure 1.

A total of 51 articles were included, as the authors of these had defined the number of phases and at least some of the parameters of the scanning protocol. These articles are listed chronologically in order of most recently published in Table 1. In the case of six studies, the authors defined two different protocols, thus amounting to 56 scanning protocols. These alternative protocols were added in Table 1 as secondary rows in relation to the first protocol.

3.2. Characteristics of the Included Studies. The peerreviewed literature on the subject of $4 \mathrm{DCT}$ for the detection of parathyroid adenomas is recent: the concept of 4DCT for the detection of parathyroid adenomas was coined in 2006 by Rodgers et al. Among the included studies, $45 \%$ were published in the last five years. Most studies (76.5\%) are retrospective studies.

3.3. Number of Phases. Most authors use a triphasic approach: 25 study protocols were described in this manner. A four-phasic approach is almost as prevalent; 23 study protocols were described in this manner. Far less common are biphasic studies: only 7 of these protocols could be identified. One study was unique since the authors made use of a five-phasic approach.

3.4. Use of a Noncontrast Enhanced Phase. Fifty-two out of 56 study protocols include a noncontrast enhanced phase (NECT), before administration of contrast medium. Only 4 protocols were identified in which the authors did not acquire a NECT; these protocols were all two-phasic in nature.

3.5. Contrast Phase Timing and Bolus Tracking. Only three authors use bolus tracking. All other authors opt for a fixed time interval after the start of contrast bolus administration before obtaining the first contrast phase, almost always an arterial phase.

Many authors do not provide definitions for the later phases in absolute terms (relation to the start of contrast bolus administration) and refer to relative intervals between the different phases. Since many authors do not describe pitch and rotation speed or refer to an exact scan length, the scan time of the different phases themselves is often unclear. This further complicates the definition of an exact timeframe. We expressed (where possible) all times in absolute relation to the onset of contrast administration; some error can be expected on a conversion. A graphic overview of the timing of the different phases of the selected studies is

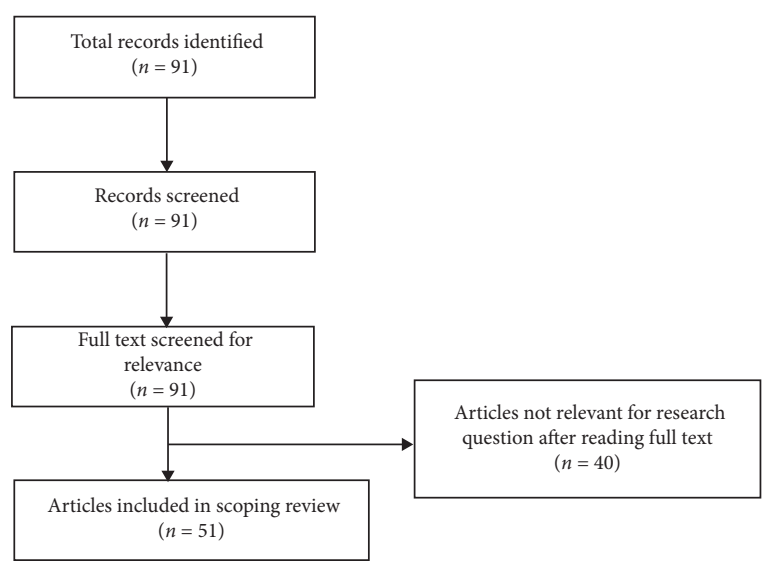

FIgURE 1: Flow chart of the studies' selection process.

presented in Figure 2; this is in relation to the number of protocols citing these relevant time points.

3.6. Use of an Arterial Phase. Except for four studies, all cited studies choose to obtain an arterial phase. In the case of 25 different studies, the arterial images were obtained $25 \mathrm{~s}$ after contrast administration; in 13 studies, the authors proposed scanning after $30 \mathrm{~s}$. Five other authors stated that the arterial images were obtained by scanning after 25-30 s. Forty-two protocols then perform arterial scanning after 25 to $30 \mathrm{~s}$.

3.7. Use of Other Phases. The venous phase is obtained most often and can be acquired as early as 45 seconds up to 70 seconds after administration of contrast. The delayed venous phase is less popular and is usually acquired between 70 seconds and as late as 90 seconds after administration of contrast. Rarely, authors also acquire an even more delayed phase: later than 100 seconds after bolus administration and even up to 130 seconds.

3.8. Contrast Administration. Most studies define the used contrast medium, bolus volume, and injection speed. A mean volume of $100 \mathrm{~mL}$ contrast is administered, most commonly at 3 or $4 \mathrm{~mL} / \mathrm{s}$. Two authors define the contrast volume in function of the patient's weight.

3.9. Effective Dose. Only 24 authors present data concerning the effective dose. The described doses are wide-ranging, with the lowest dose reported at $5.5 \mathrm{mSv}$ and an upper limit of $28 \mathrm{mSv}$. The mean effective dose for all relevant studies combined is calculated at $15.96 \mathrm{mSv}$. 27 authors do not discuss dose limits.

The mean effective dose for the three biphasic studies with available data is calculated at $8.1 \mathrm{mSv}$. For the three- and fourphasic studies, the mean effective dose is higher, $18.1 \mathrm{mSv}$ and $15.4 \mathrm{mSv}$, respectively. The authors of the only five-phasic study report an effective dose range of 5.6-10.4 mSv.

3.10. Appraisal of Sensitivity/Specificity. All included studies were assessed for the quality of the reported findings. 74.5\% of the included studies report on sensitivity and specificity. 


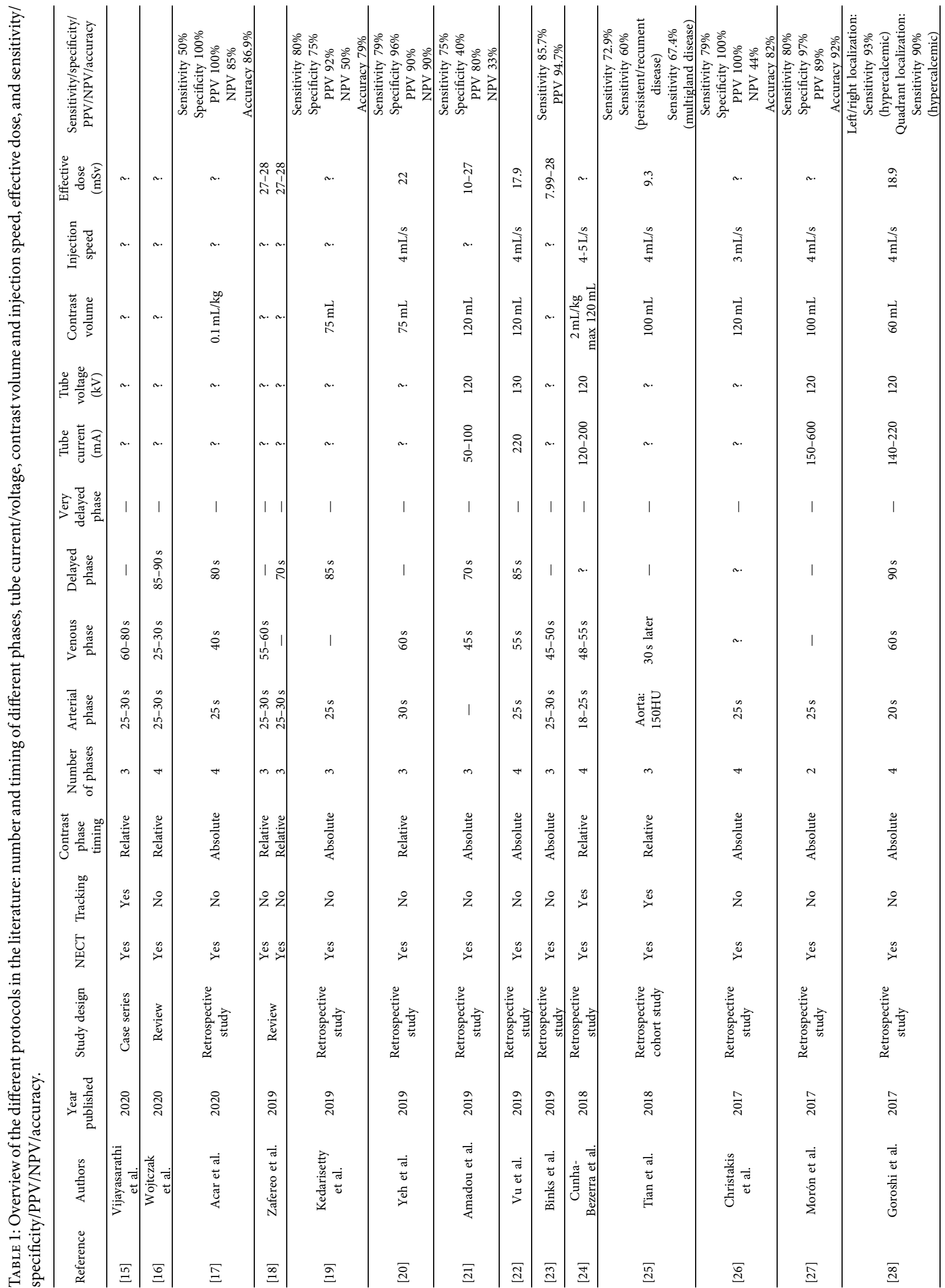




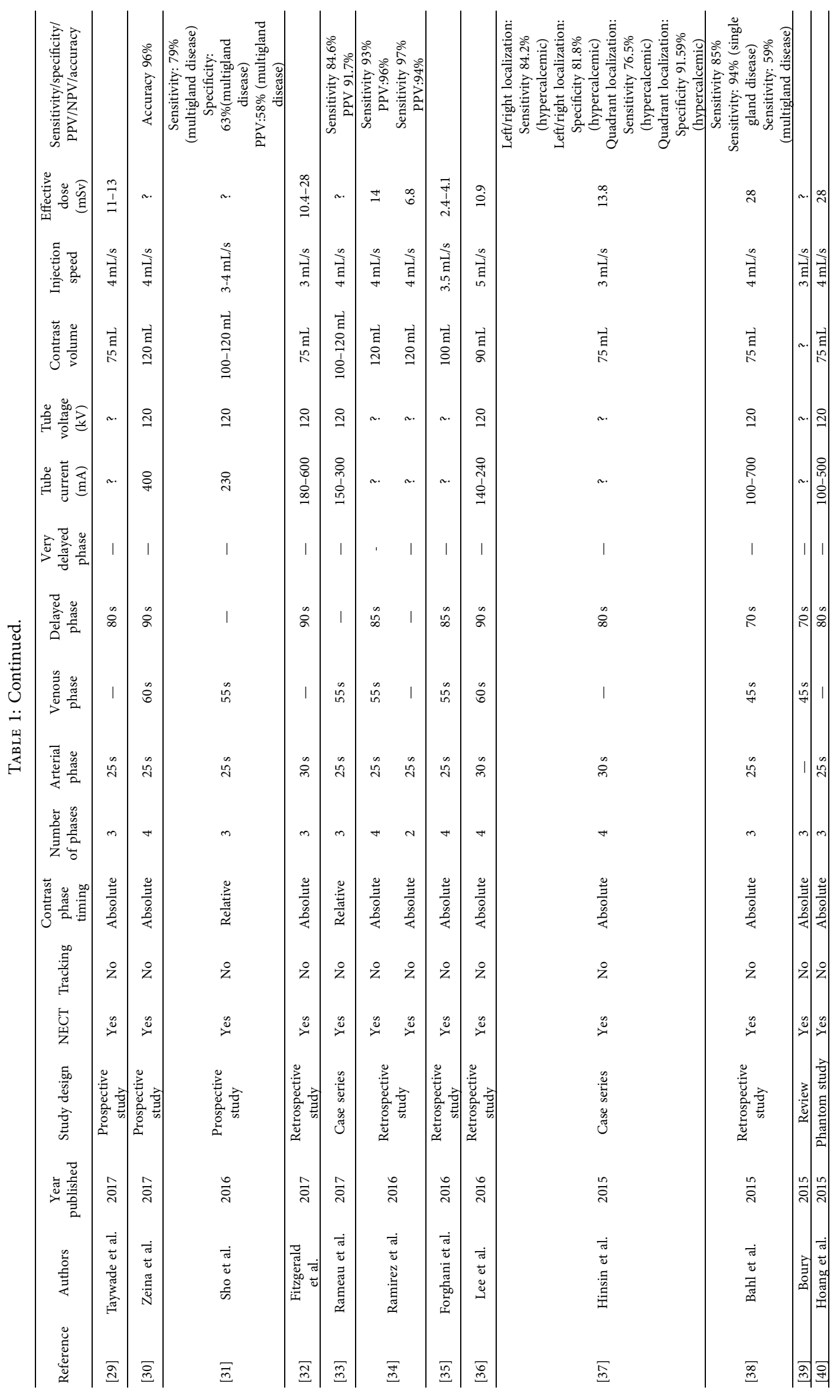




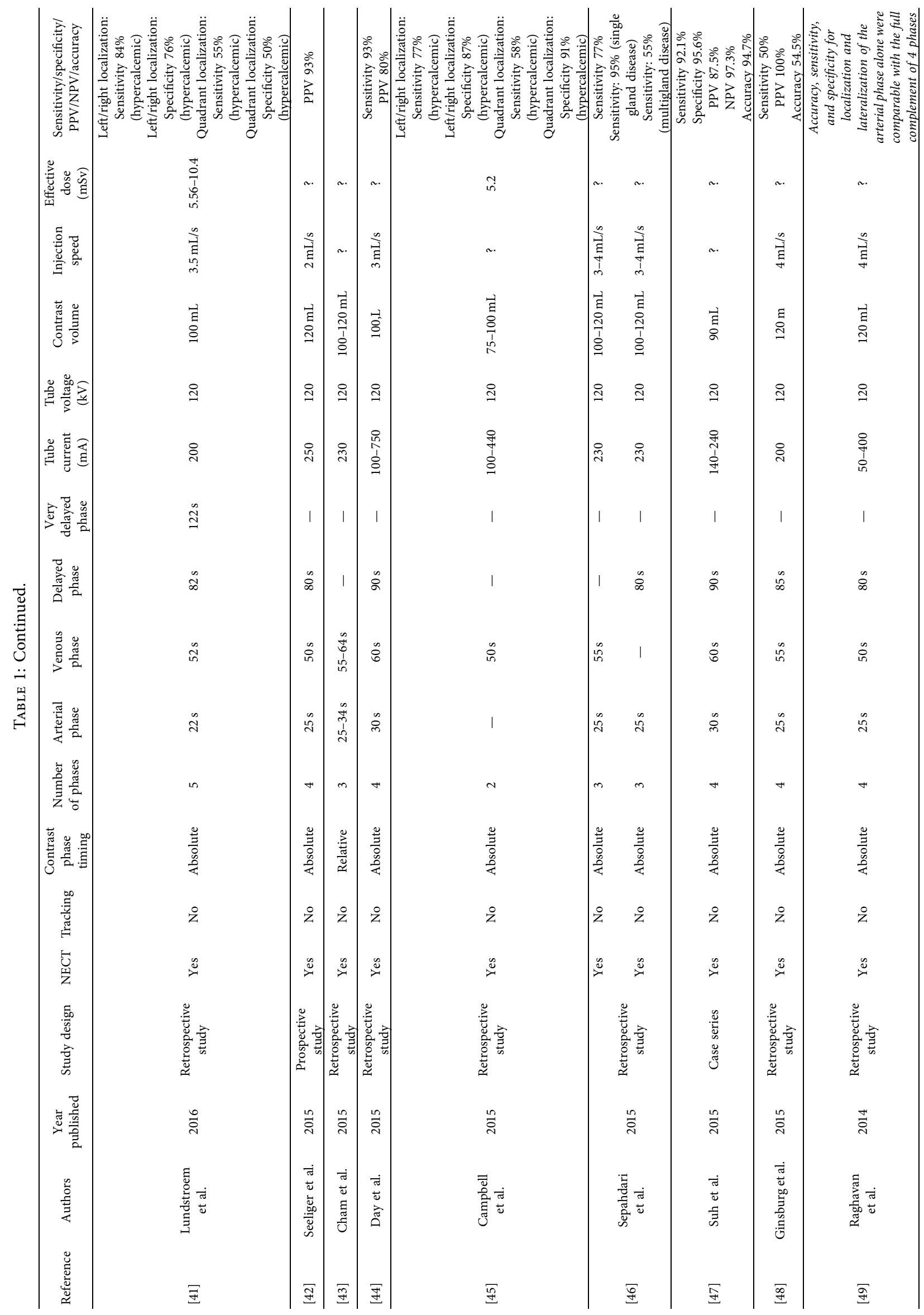




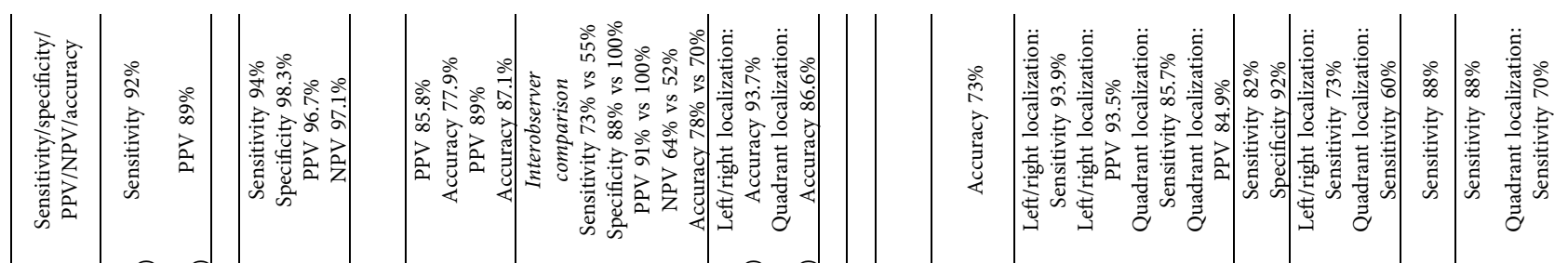

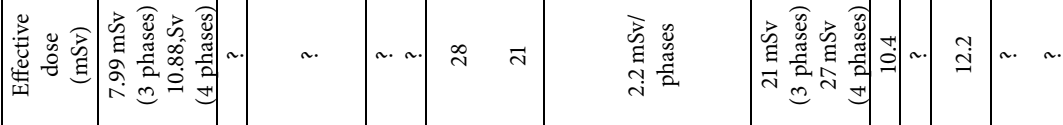

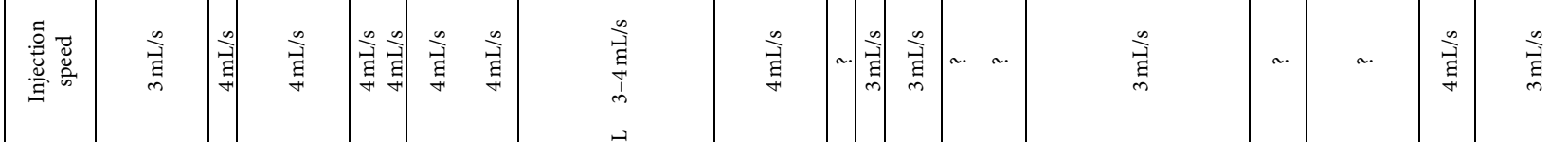

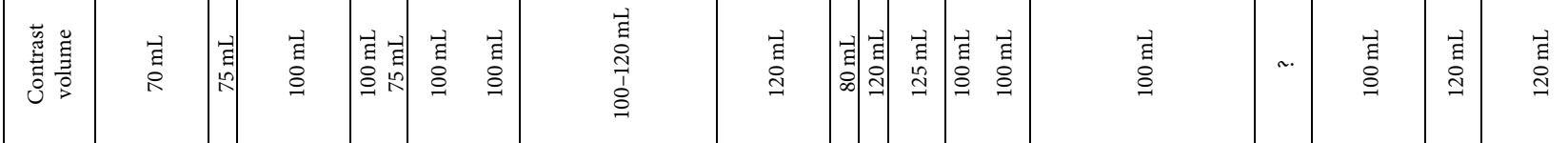

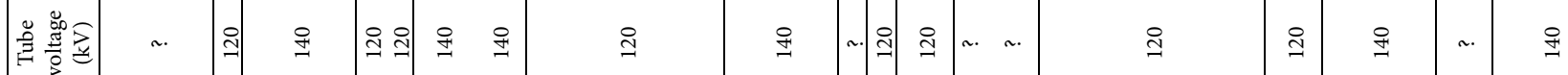

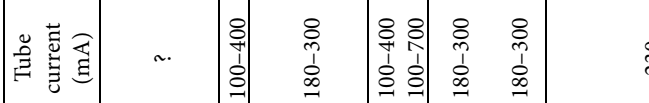

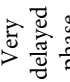

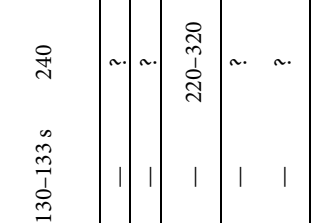

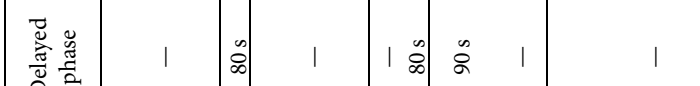

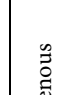

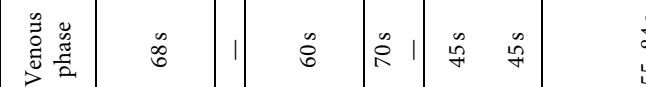

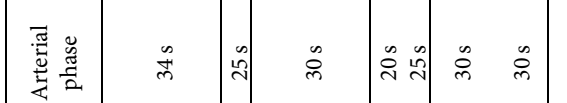

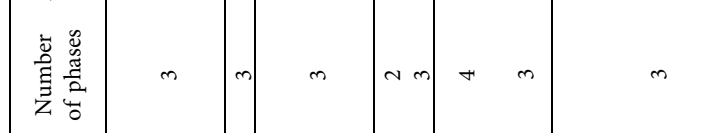

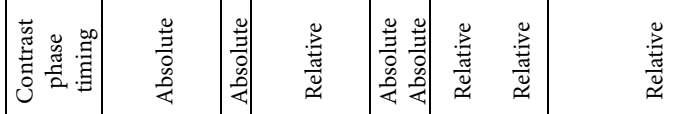
(1) (1)

(2)

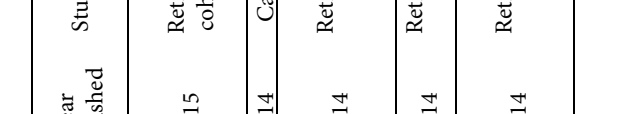

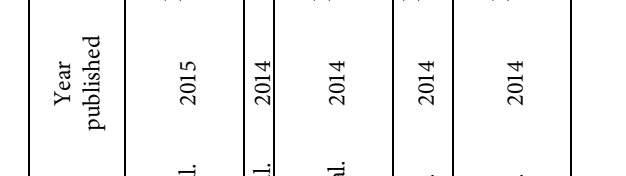

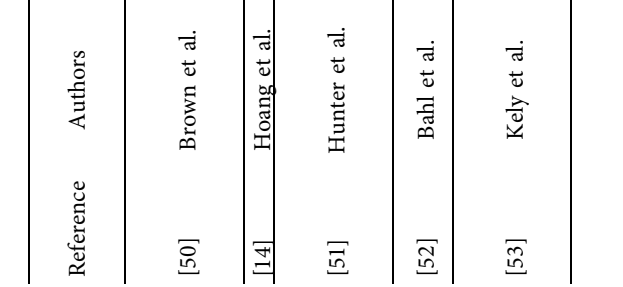

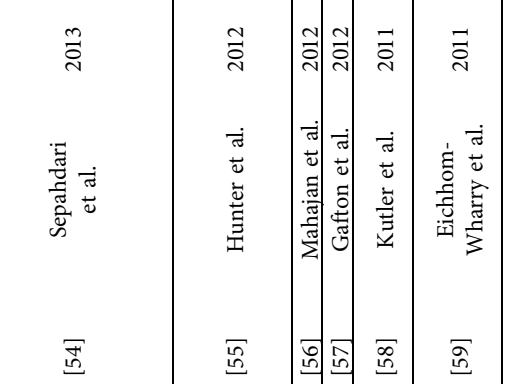

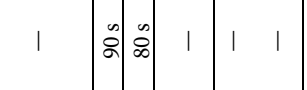

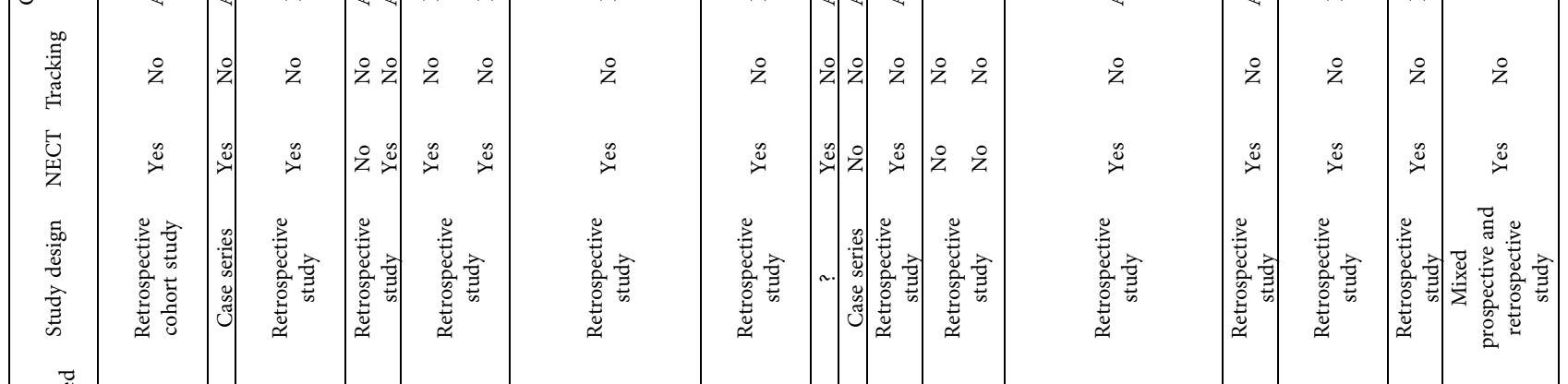
西

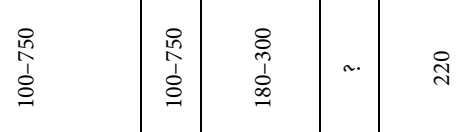
$\stackrel{\leftrightarrow}{\Im}$

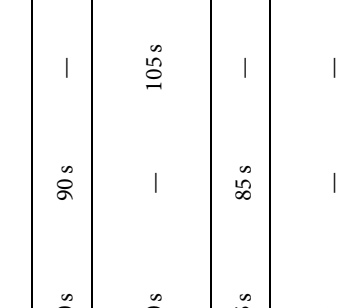

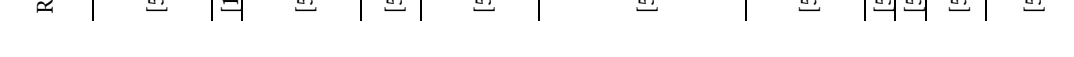




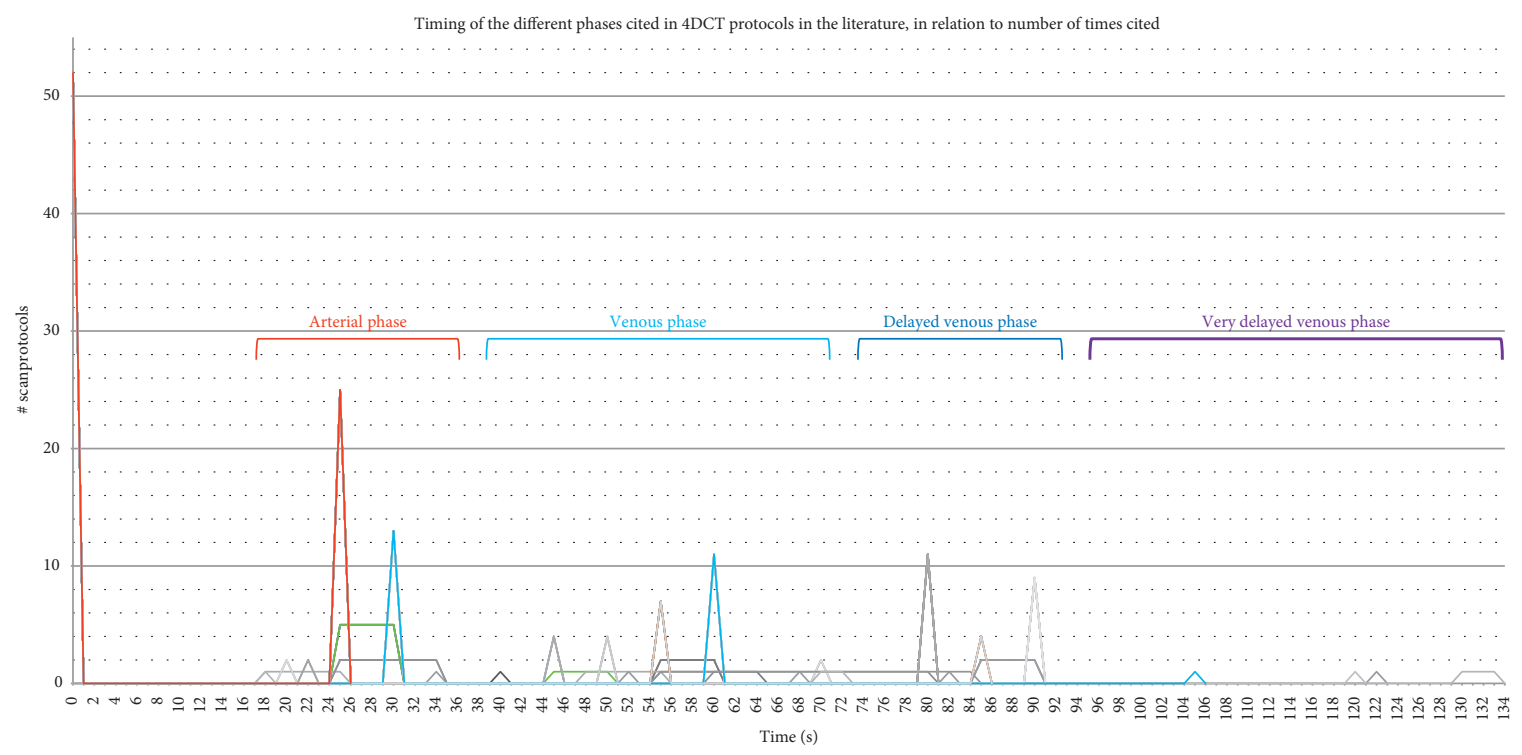

Figure 2: Timing of the different phases of 4DCT protocols in the literature, in relation to the number of citations for each of these relevant time points.

Some studies (14\%) do not refer to general sensitivity but report on the sensitivity of lateralization or correct selection of the diseased quadrant.

We find a mean sensitivity $(n=23)$ of $81.5 \%$ for the detection of parathyroid adenomas using 4DCT. Only two studies report a lower sensitivity of 50\%; both studies are four-phasic studies. We find a mean PPV $(n=17)$ of $90 \%$. Less data is available on specificity $(n=11)$, and the mean specificity is calculated at $86 \%$.

\section{Discussion}

Typical parathyroid adenomas are hypoattenuating to thyroid tissue on NECT and demonstrate avid arterial enhancement during the arterial phase as well as rapid washout on the venous phase. The arterial phase is the most important, as adenomas can be visualized as hyperenhancing nodules in characteristic locations. In Figure 3, we provide an example of a parathyroid adenoma behaving accordingly during all three phases. Unfortunately, for approximately one-third of adenomas, the lesion will prove to be isoattenuating to the thyroid on arterial and venous phases [15].

Most authors agree to include a noncontrast enhanced phase. This phase does seem to be important in the diagnosis of parathyroid adenomas, as the study by Bahl et al. [38] identifies a type $\mathrm{C}$ pattern of enhancement. These lesions mimic thyroid tissue and can only be discerned by the use of noncontrast-enhanced imaging since these lesions should be lower in density compared to the thyroid on the noncontrast enhanced series. Almost all authors choose to include an arterial phase. According to Bahl et al., this is necessary to diagnose type A lesions, which demonstrate a higher peak enhancement compared to the thyroid in the arterial phase. A venous or delayed venous phase is commonly used.
According to Bahl et al., this is necessary to diagnose type B lesions, which demonstrate a lower enhancement compared to the thyroid in the venous phase.

Most authors opt for a delay of 25 up to 30 seconds for the arterial phase after contrast administration. There is more variance concerning the timing of the venous phases, with some authors scanning as early as 40 seconds after administration of contrast and others no less than $50 \mathrm{sec}$ onds later. Other authors even prefer very delayed venous phases. We find no difference regarding the sensitivity or specificity of 4DCT in comparison to the number of phases or their timing. The two studies with the lowest sensitivity are both four-phasic studies, with numerous three-phasic studies reporting higher sensitivity.

Only three authors use bolus tracking to optimize the scanning delay, although this could easily be implemented. If the arterial peak enhancement of the adenoma should prove to be a short-lived effect, scanning at a fixed interval of time might risk missing the diagnosis. This would prove especially true in older patients with suboptimal cardiac output, as the arterial phase could be performed too early in these cases. Bolus tracking at the level of the aortic arch would allow for the arterial and later phases to be obtained at a variable time interval, with reduced variability of arterial enhancement and less risk of scanning prematurely. Bolus tracking is commonly used in clinical practice, without significant dose effect [64].

Regarding contrast administration, the variance between the reviewed studies is limited. Administered bolus volumes are situated between $75 \mathrm{~mL}$ and $120 \mathrm{~mL}$ with a mean volume of $100 \mathrm{~mL}$. Injection speed varies mostly between 3 and $4 \mathrm{~mL} / \mathrm{s}$. A contrast bolus with a lower volume or at a lower injection speed may affect lesion enhancement and the timing of said enhancement. No difference regarding sensitivity or specificity of $4 \mathrm{DCT}$ is found, however, for different volumes or injection speeds. 


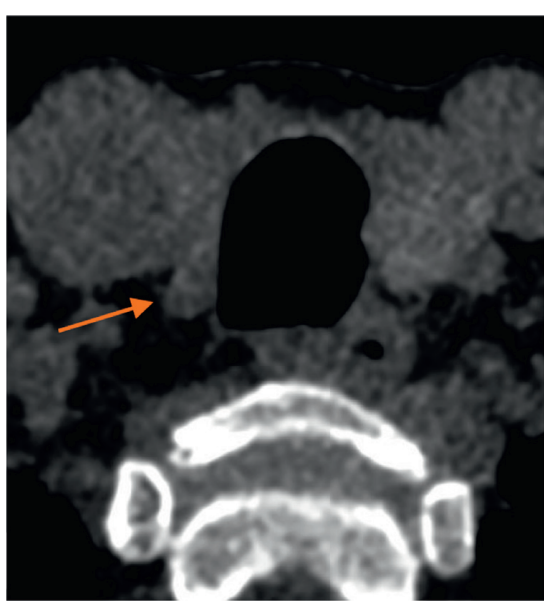

(a)

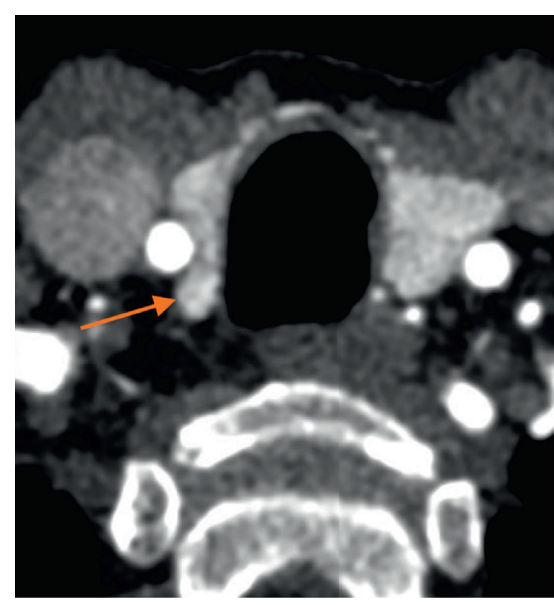

(b)

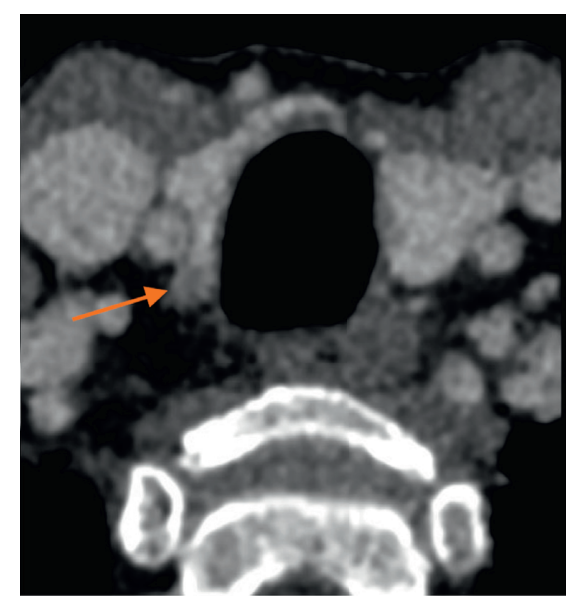

(c)

FIGURE 3: 4DCT of a small right upper parathyroid adenoma. (a) NECT with lower attenuation (b) Arterial phase with higher enhancement. (c) Venous phase with lower enhancement.

What proves striking is that 27 out of 51 authors do not mention effective dose when discussing a multiphasic CT scan of the neck. The mean effective dose for the 24 studies that do comment on patient dose is calculated at $15.96 \mathrm{mSv}$. The described dose limits are wide-ranging, with an upper limit of $28 \mathrm{mSv}$. These upper values should be considered as very high and place direct limits on the number of phases. As a limitation, it should be noted that it is difficult to calculate a correct effective organ dose. The presence of an iodinated contrast medium is a known factor that increases organ dose, yet this has not been factored into any known conversion method [65].

The mean effective dose is the lowest for biphasic studies, as could be expected. The mean effective dose for the three studies with available effective dose data is calculated at $8.1 \mathrm{mSv}$. For the more popular three- and four-phasic studies, the mean effective dose is higher, but the mean effective dose for the four-phasic studies appears lower in comparison to the three-phasic studies $(15.4 \mathrm{mSv}$ versus $18.1 \mathrm{mSv}$ ). This is probably because of lower tube current settings, an effect that can also be observed in the only fivephasic study. Here, a low mean effective dose range of $5.6-10.4 \mathrm{mSv}$ is reported, probably because the authors do not use automatic tube current modulation but instead opt for a fixed tube current of $200 \mathrm{~mA}$.

Changes in tube current will affect image noise: higher $\mathrm{mA}$ settings will result in lower noise. Changes in tube voltage will affect the amount of contrast in the resultant image: higher $\mathrm{kV}$ settings will result in an increase in contrast. Since increasing the tube current improves image quality by reducing noise without affecting image contrast, it can be argued that to keep the effective dose for classic 4DCT within acceptable levels, a lower fixed tube current should be preferred. Most protocols utilize a $120 \mathrm{kV}$ tube voltage. Since diagnosis relies primarily on enhancement after contrast, it can be suggested that scanning at $100 \mathrm{kV}$ tube voltage would yield similar image contrast, but at a lower effective dose [66]. Again, regarding tube parameters, no difference in sensitivity or specificity is found for different values.

\section{Conclusion}

Many different 4DCT scanning protocols for the detection of parathyroid adenomas exist in the literature. We find a mean sensitivity of $81.5 \%$ and a mean specificity of $86 \%$. The number of phases does not appear to affect sensitivity or specificity. A triphasic approach, however, seems preferable, as three patterns of enhancement of parathyroid adenomas are described and require nonenhanced imaging as well as an arterial and a venous series. Arterial images are usually obtained 25-30s after administration of contrast, and less agreement exists concerning the timing of the venous phase(s). A mean contrast bolus of $100 \mathrm{~mL}$ is administered at $3-4 \mathrm{~mL} / \mathrm{s}$. Bolus tracking is not often used but could help to reduce the variability of enhancement. Sensitivity and specificity do not appear to be affected by other scan parameters like tube voltage or tube current. To keep the effective dose within limits, scanning at a lower fixed tube current seems preferable. Lowering tube voltage from $120 \mathrm{kV}$ to $100 \mathrm{kV}$ may yield similar image contrast but would also help lower the dose.

\section{Data Availability}

No data were used to support this study.

\section{Conflicts of Interest}

The authors declare that they have no conflicts of interest.

\section{Authors' Contributions}

SR reviewed the literature and wrote the text. MT revised the final manuscript.

\section{References}

[1] J. P. Bilezikian, M. L. Brandi, R. Eastell et al., "Guidelines for the management of asymptomatic primary hyperparathyroidism: summary statement from the Fourth International 
Workshop," The Journal of Clinical Endocrinology \& Metabolism, vol. 99, no. 10, pp. 3561-3569, 2014.

[2] S. J. Silverberg, B. L. Clarke, M. Peacock et al., "Current issues in the presentation of asymptomatic primary hyperparathyroidism: proceedings of the Fourth International Workshop," The Journal of Clinical Endocrinology \& Metabolism, vol. 99, no. 10, pp. 3580-3594, 2014.

[3] R. Udelsman, G. Åkerström, C. Biagini et al., "The surgical management of asymptomatic primary hyperparathyroidism: proceedings of the Fourth International Workshop," The Journal of Clinical Endocrinology \& Metabolism, vol. 99, no. 10, pp. 3595-3606, 2014.

[4] S. Y. Morita, H. Somervell, C. B. Umbricht, A. P. B. Dackiw, and M. A. Zeiger, "Evaluation for concomitant thyroid nodules and primary hyperparathyroidism in patients undergoing parathyroidectomy or thyroidectomy," Surgery, vol. 144 , no. 6 , pp. 862-867, 2008.

[5] G. M. Vitetta, P. Neri, A. Chiecchio et al., "Role of ultrasonography in the management of patients with primary hyperparathyroidism: retrospective comparison with technetium-99m sestamibi scintigraphy," Journal of Ultrasound, vol. 17, no. 1, pp. 1-12, 2014.

[6] Z. Devcic, R. B. Jeffrey, A. Kamaya, and T. S. Desser, "The elusive parathyroid adenoma," Ultrasound Quarterly, vol. 29, no. 3, pp. 179-187, 2013.

[7] E. Berber, R. T. Parikh, N. Ballem, C. N. Garner, M. Milas, and A. E. Siperstein, "Factors contributing to negative parathyroid localization: an analysis of 1000 patients," Surgery, vol. 144, no. 1, pp. 74-79, 2008.

[8] C. Guerin, A. Lowery, S. Gabriel et al., "Preoperative imaging for focused parathyroidectomy: making a good strategy even better," European Journal of Endocrinology, vol. 172, no. 5, pp. 519-526, 2015.

[9] E. Hindié, P. Zanotti-Fregonara, A. Tabarin et al., "The role of radionuclide imaging in the surgical management of primary hyperparathyroidism," Journal of Nuclear Medicine, vol. 56, no. 5, pp. 737-744, 2015.

[10] E. L. Hänninen, T. J. Vogl, T. Steinmüller, J. Ricke, P. Neuhaus, and R. Felix, "Preoperative contrast-enhanced MRI of the parathyroid glands in hyperparathyroidism," Investigative Radiology, vol. 35, no. 7, pp. 426-430, 2000.

[11] A. M. Grayev, L. R. Gentry, M. J. Hartman, H. Chen, S. B. Perlman, and S. B. Reeder, "Presurgical localization of parathyroid adenomas with magnetic resonance imaging at 3.0 T: an adjunct method to supplement traditional imaging," Annals of Surgical Oncology, vol. 19, no. 3, pp. 981-989, 2012.

[12] B. Sacconi, R. Argirò, D. Diacinti et al., "MR appearance of parathyroid adenomas at $3 \mathrm{~T}$ in patients with primary hyperparathyroidism: what radiologists need to know for preoperative localization," European Radiology, vol. 26, no. 3, pp. 664-673, 2016.

[13] L. F. Starker, A. Mahajan, P. Björklund, G. Sze, R. Udelsman, and T. Carling, "4D parathyroid CT as the initial localization study for patients with de novo primary hyperparathyroidism," Annals of Surgical Oncology, vol. 18, no. 6, pp. 1723-1728, 2011.

[14] J. K. Hoang, W.-k. Sung, M. Bahl, and C. D. Phillips, "How to perform parathyroid $4 \mathrm{D}$ CT: tips and traps for technique and interpretation," Radiology, vol. 270, no. 1, pp. 15-24, 2014.

[15] A. Vijayasarathi, S. Karnezis, A. Azizyan, N. Salamon, and A. Sepahdari, "4D CT parathyroid for the general radiologist: a pictorial essay of illustrative cases," Current Problems in Diagnostic Radiology, vol. 20, pp. 30046-30053, 2020.

[16] B. Wojtczak, J. Syrycka, K. Kaliszewski, J. Rudnicki, M. Bolanowski, and M. Barczyński, "Surgical implications of recent modalities for parathyroid imaging," Gland Surgery, vol. 9, no. 2, pp. S86-S94, 2020.

[17] N. Acar, M. Haciyanli, M. Coskun et al., "Diagnostic value of four-dimensional computed tomography and four-dimensional magnetic resonance imaging in primary hyperparathyroidism when first-line imaging was inadequate," The Annals of The Royal College of Surgeons of England, vol. 102, no. 4, pp. 294-299, 2020.

[18] M. Zafereo, J. Yu, P. Angelos et al., "American head and neck society endocrine surgery section update on parathyroid imaging for surgical candidates with primary hyperparathyroidism," Head \& Neck, vol. 41, no. 7, pp. 2398-2409, 2019.

[19] S. Kedarisetty, C. Fundakowski, K. Ramakrishnan, and S. Dadparvar, "Clinical value of Tc99m-MIBI SPECT/CT versus $4 \mathrm{D}-\mathrm{CT}$ or US in management of patients with hyperparathyroidism," Ear, Nose \& Throat Journal, vol. 98, no. 3, pp. 149-157, 2019.

[20] R. Yeh, Y.-K. D. Tay, G. Tabacco et al., "Diagnostic performance of $4 \mathrm{D}$ CT and sestamibi SPECT/CT in localizing parathyroid adenomas in primary hyperparathyroidism," Radiology, vol. 291, no. 2, pp. 469-476, 2019.

[21] C. Amadou, G. Bera, M. Ezziane et al., "18F-fluorocholine $\mathrm{PET} / \mathrm{CT}$ and parathyroid 4D computed tomography for primary hyperparathyroidism: the challenge of reoperative patients," World Journal of Surgery, vol. 43, no. 5, pp. 1232-1242, 2019.

[22] T. H. Vu, D. Schellingerhout, N. Guha-Thakurta et al., "Solitary parathyroid adenoma localization in technetium Tc99m Sestamibi SPECT and multiphase multidetector 4D CT," American Journal of Neuroradiology, vol. 40, no. 1, pp. 142-149, 2019.

[23] M. Binks, D. Burrows, and D. Littlejohn, “A rural perspective on minimally invasive parathyroidectomy: optimal preoperative imaging and patient outcomes," ANZ Journal of Surgery, vol. 89, no. 1-2, pp. 43-47, 2019.

[24] P. Cunha-Bezerra, R. Vieira, F. Amaral, H. Cartaxo, T. Lima, and U. Montarroyos, "Better performance of four-dimension computed tomography as a localization procedure in normocalcemic primary hyperparathyroidism," Journal of Medical Imaging and Radiation Oncology, vol. 15, 2018.

[25] Y. Tian, S. T. Tanny, P. Einsiedel et al., "Four-dimensional computed tomography: clinical impact for patients with primary hyperparathyroidism," Annals of Surgical Oncology, vol. 25, no. 1, pp. 117-121, 2018.

[26] I. Christakis, T. Vu, H. H. Chuang et al., "The diagnostic accuracy of neck ultrasound, 4D-Computed tomographyand sestamibi imaging in parathyroid carcinoma," European Journal of Radiology, vol. 95, pp. 82-88, 2017.

[27] F. Morón, A. Delumpa, J. Chetta, D. Guffey, and D. Dunaway, "Single phase computed tomography is equivalent to dual phase method for localizing hyperfunctioning parathyroid glands in patients with primary hyperparathyroidism: a retrospective review," PeerJ, vol. 5, Article ID e3586, 2017.

[28] M. Goroshi, A. R. Lila, S. S. Jadhav et al., "Percentage arterial enhancement: an objective index for accurate identification of parathyroid adenoma/hyperplasia in primary hyperparathyroidism," Clinical Endocrinology, vol. 87, no. 6, pp. 791-798, 2017.

[29] S. K. Taywade, N. A. Damle, A Behera et al., "Comparison of $18 \mathrm{~F}$-fluorocholine positron emission tomography/computed tomography and four-dimensional computed tomography in the preoperative localization of parathyroid adenomas-initial results," Indian Journal of Endocrinology and Metabolism, vol. 21, no. 3, pp. 399-403, 2017. 
[30] A. R. Zeina, H. Nakar, N Reindorp D et al., "Four-dimensional computed tomography (4DCT) for preoperative localization of parathyroid adenomas," The Israel Medical Association Journal: IMAJ, vol. 19, no. 4, pp. 216-220, 2017.

[31] S. Sho, M. Yilma, M. W. Yeh et al., "Prospective validation of two 4D-CT-based scoring systems for prediction of multigland disease in primary hyperparathyroidism," American Journal of Neuroradiology, vol. 37, no. 12, pp. 2323-2327, 2016.

[32] R. T. Fitzgerald, M. Kuruva, R. David et al., "Characterization of thyroid nodules by 4-dimensional computed tomography," Journal of Computer Assisted Tomography, vol. 41, no. 2, pp. 195-198, 2017.

[33] A. Rameau, S. Eng, J. Vu, R. Saket, P. Jun, and M. Friduss, "Four-dimensional computed tomography scan utility in parathyroidectomy for primary hyperparathyroidism with low baseline intact parathyroid hormone," The Laryngoscope, vol. 127, no. 6, pp. 1476-1482, 2017.

[34] A. G. Ramirez, A. L. Shada, A. N. Martin et al., "Clinical efficacy of 2-phase versus 4-phase computed tomography for localization in primary hyperparathyroidism," Surgery, vol. 160 , no. 3, pp. 731-737, 2016.

[35] R. Forghani, M. Roskies, X. Liu et al., "Dual-energy CT characteristics of parathyroid adenomas on 25-and 55-second 4D-CT acquisitions," Journal of Computer Assisted Tomography, vol. 40, no. 5, pp. 806-814, 2016.

[36] E. K. Lee, T. J. Yun, J.-h. Kim et al., "Effect of tumor volume on the enhancement pattern of parathyroid adenoma on parathyroid four-dimensional CT," Neuroradiology, vol. 58, no. 5, pp. 495-501, 2016.

[37] A. M. Hinson, D. R. Lee, B. A. Hobbs, R. T. Fitzgerald, D. L. Bodenner, and B. C. Stack, "Preoperative 4D CT localization of nonlocalizing parathyroid adenomas by ultrasound and SPECT-CT," Otolaryngology-Head and Neck Surgery, vol. 153, no. 5, pp. 775-778, 2015.

[38] M. Bahl, A. R. Sepahdari, J. A. Sosa, and J. K. Hoang, "Parathyroid adenomas and hyperplasia on four-dimensional CT scans: three patterns of enhancement relative to the thyroid gland justify a three-phase protocol," Radiology, vol. 277, no. 2, pp. 454-462, 2015.

[39] S. Boury, "New methods for parathyroid imaging: sonography, 4D CT, MRI," Annales d'Endocrinologie, vol. 76, no. 2, pp. 148-152, 2015.

[40] J. K. Hoang, R. E. Reiman, G. B. Nguyen et al., "Lifetime attributable risk of cancer from radiation exposure during parathyroid imaging: comparison of 4D CT and parathyroid scintigraphy," American Journal of Roentgenology, vol. 204, no. 5, pp. W579-W585, 2015.

[41] A. K. Lundstroem, W. Trolle, C. H. Soerensen, and P. S. Myschetzky, "Preoperative localization of hyperfunctioning parathyroid glands with 4D-CT," European Archives of Oto-Rhino-Laryngology, vol. 273, no. 5, pp. 1253-1259, 2016.

[42] B. Seeliger, P. F. Alesina, J.-A. Koch, J. Hinrichs, B. Meier, and M. K. Walz, "Diagnostic value and clinical impact of complementary CT scan prior to surgery for non-localized primary hyperparathyroidism," Langenbeck's Archives of Surgery, vol. 400, no. 3, pp. 307-312, 2015.

[43] S. Cham, A. R. Sepahdari, K. E. Hall, M. W. Yeh, and A. Harari, "Dynamic parathyroid computed tomography (4DCT) facilitates reoperative parathyroidectomy and enables cure of missed hyperplasia," Annals of Surgical Oncology, vol. 22, no. 11, pp. 3537-3542, 2015.
[44] K. M. Day, M. Elsayed, M. D. Beland, and J. M. Monchik, "The utility of 4-dimensional computed tomography for preoperative localization of primary hyperparathyroidism in patients not localized by sestamibi or ultrasonography," Surgery, vol. 157, no. 3, pp. 534-539, 2015.

[45] M. J. Campbell, P. Sicuro, A. Alseidi, C. C. Blackmore, and J. A. Ryan, "Two-phase (low-dose) computed tomography is as effective as $4 \mathrm{D}$-CT for identifying enlarged parathyroid glands," International Journal of Surgery, vol. 14, pp. 80-84, 2015.

[46] A. R. Sepahdari, M. Bahl, A. Harari, H. J. Kim, M. W. Yeh, and J. K. Hoang, "Predictors of multigland disease in primary hyperparathyroidism: a scoring system with $4 \mathrm{D}-\mathrm{CT}$ imaging and biochemical markers," American Journal of Neuroradiology, vol. 36, no. 5, pp. 987-992, 2015.

[47] Y. J. Suh, J. Y. Choi, S.-j. Kim et al., "Comparison of 4D CT, ultrasonography, and 99mTc sestamibi SPECT/CT in localizing single-gland primary hyperparathyroidism," Otolaryngology-Head and Neck Surgery, vol. 152, no. 3, pp. 438-443, 2015.

[48] M. Ginsburg, G. A. Christoforidis, S. P. Zivin et al., "Adenoma localization for recurrent or persistent primary hyperparathyroidism using dynamic four-dimensional CT and venous sampling," Journal of Vascular and Interventional Radiology, vol. 26, no. 1, pp. 79-86, 2015.

[49] P. Raghavan, C. R. Durst, D. A. Ornan et al., "Dynamic CT for parathyroid disease: are multiple phases necessary?" American Journal of Neuroradiology, vol. 35, no. 10, pp. 1959-1964, 2014.

[50] S. J. Brown, J. C. Lee, J. Christie et al., "Four-dimensional computed tomography for parathyroid localization: a new imaging modality," ANZ Journal of Surgery, vol. 85, no. 6, pp. $483-487,2015$.

[51] G. J. Hunter, D. T. Ginat, H. R. Kelly, E. F. Halpern, and L. M. Hamberg, "Discriminating parathyroid adenoma from local mimics by using inherent tissue attenuation and vascular information obtained with four-dimensional CT: formulation of a multinomial logistic regression model," Radiology, vol. 270, no. 1, pp. 168-175, 2014.

[52] M. Bahl, M. Muzaffar, G. Vij, J. A. Sosa, K. R. Choudhury, and J. K. Hoang, "Prevalence of the polar vessel sign in parathyroid adenomas on the arterial phase of $4 \mathrm{D}$ CT," American Journal of Neuroradiology, vol. 35, no. 3, pp. 578-581, 2014.

[53] H. R. Kelly, L. M. Hamberg, and G. J. Hunter, “4D-CT for preoperative localization of abnormal parathyroid glands in patients with hyperparathyroidism: accuracy and ability to stratify patients by unilateral versus bilateral disease in surgery-naïve and re-exploration patients," American Journal of Neuroradiology, vol. 35, no. 1, pp. 176-181, 2014.

[54] A. R. Sepahdari, M. W. Yeh, D. Rodrigues, S. N. Khan, and A. Harari, "Three-phase parathyroid 4-dimensional computed tomography initial experience," Journal of Computer Assisted Tomography, vol. 37, no. 4, pp. 511-517, 2013.

[55] G. J. Hunter, D. Schellingerhout, T. H. Vu, N. D. Perrier, and L. M. Hamberg, "Accuracy of four-dimensional CT for the localization of abnormal parathyroid glands in patients with primary hyperparathyroidism," Radiology, vol. 264, no. 3, pp. 789-795, 2012.

[56] A. Mahajan, L. F. Starker, M. Ghita, R. Udelsman, J. A. Brink, and T. Carling, "Parathyroid four-dimensional computed tomography: evaluation of radiation dose exposure during preoperative localization of parathyroid tumors in primary hyperparathyroidism," World Journal of Surgery, vol. 36, no. 6, pp. 1335-1339, 2012. 
[57] A. R. Gafton, C. M. Glastonbury, J. D. Eastwood, and J. K. Hoang, "Parathyroid lesions: characterization with dualphase arterial and venous enhanced CT of the neck," American Journal of Neuroradiology, vol. 33, no. 5, pp. 949952, 2012.

[58] D. I. Kutler, R. Moquete, E. Kazam, and W. I. Kuhel, "Parathyroid localization with modified 4D-computed tomography and ultrasonography for patients with primary hyperparathyroidism," The Laryngoscope, vol. 121, no. 6, pp. 1219-1224, 2011

[59] L. I. Eichhorn-Wharry, A. M. Carlin, and G. B. Talpos, "Mild hypercalcemia: an indication to select 4-dimensional computed tomography scan for preoperative localization of parathyroid adenomas," The American Journal of Surgery, vol. 201, no. 3, pp. 334-338, 2011.

[60] M. D. Beland, W. W. Mayo-Smith, D. J. Grand, J. T. Machan, and J. M. Monchik, "Dynamic MDCT for localization of occult parathyroid adenomas in 26 patients with primary hyperparathyroidism," American Journal of Roentgenology, vol. 196, no. 1, pp. 61-65, 2011.

[61] C. C. Lubitz, G. J. Hunter, L. M. Hamberg et al., "Accuracy of 4-dimensional computed tomography in poorly localized patients with primary hyperparathyroidism," Surgery, vol. 148, no. 6, pp. 1129-1138, 2010.

[62] M. M. Mortenson, D. B. Evans, J. E. Lee et al., "Parathyroid exploration in the reoperative neck: improved preoperative localization with 4D-computed tomography," Journal of the American College of Surgeons, vol. 206, no. 5, pp. 888-895, 2008.

[63] S. E. Rodgers, G. J. Hunter, L. M. Hamberg et al., "Improved preoperative planning for directed parathyroidectomy with 4dimensional computed tomography," Surgery, vol. 140, no. 6, pp. 932-941, 2006.

[64] D. P. Frush, E. B. Spencer, L. F. Donnelly, J. Y. Zheng, D. M. DeLong, and G. S. Bisset, "Optimizing contrast-enhanced abdominal CT in infants and children using bolus tracking," American Journal of Roentgenology, vol. 172, no. 4, pp. 1007-1013, 1999.

[65] M. Mazloumi, G. Van Gompel, V. Kersemans, J. de Mey, and N. Buls, "The presence of contrast agent increases organ radiation dose in contrast-enhanced CT," European Radiology, vol. 30, 2021.

[66] M. K. Gill, A. Vijayananthan, G. Kumar, K. Jayarani, K. H. Ng, and Z. Sun, "Use of $100 \mathrm{kV}$ versus $120 \mathrm{kV}$ in computed tomography pulmonary angiography in the detection of pulmonary embolism: effect on radiation dose and image quality," Quantitative Imaging in Medicine and Surgery, vol. 5, no. 4, pp. 524-533, 2015. 